\title{
Massive runaway stars in the Large Magellanic Cloud
}

\author{
V. V. Gvaramadze ${ }^{1,2,3}$, P. Kroupa ${ }^{1}$, and J. Pflamm-Altenburg ${ }^{1}$ \\ 1 Argelander-Institut für Astronomie, Universität Bonn, Auf dem Hügel 73, 53121 Bonn, Germany \\ e-mail: [pavel; jpflamm] aastro.uni-bonn.de \\ 2 Sternberg Astronomical Institute, Moscow State University, Universitetskij Pr. 13, Moscow 119992, Russia \\ e-mail: vgvaram@mx.iki.rssi.ru \\ 3 Isaac Newton Institute of Chile, Moscow Branch, Universitetskij Pr. 13, Moscow 119992, Russia
}

Received 27 April 2010/ Accepted 26 May 2010

ABSTRACT

\begin{abstract}
The origin of massive field stars in the Large Magellanic Cloud (LMC) has long been an enigma. The recent measurements of large offsets $\left(\sim 100 \mathrm{~km} \mathrm{~s}^{-1}\right)$ between the heliocentric radial velocities of some very massive (O2-type) field stars and the systemic LMC velocity provides a possible explanation of this enigma and suggests that the field stars are runaway stars ejected from their birthplaces at the very beginning of their parent cluster's dynamical evolution. A straightforward way to prove this explanation is to measure the proper motions of the field stars and to show that they are moving away from one of the nearby star clusters or $\mathrm{OB}$ associations. This approach is, however, complicated by the long distance to the LMC, which makes accurate proper motion measurements difficult. We used an alternative approach for solving the problem (first applied for Galactic field stars), based on the search for bow shocks produced by runaway stars. The geometry of detected bow shocks would allow us to infer the direction of stellar motion, thereby determining their possible parent clusters. In this paper we present the results of a search for bow shocks around six massive field stars that have been proposed as candidate runaway stars. Using archival Spitzer Space Telescope data, we found a bow shock associated with one of our programme stars, the $\mathrm{O} 2 \mathrm{~V}\left(\left(\mathrm{f}^{*}\right)\right)$ star BI 237, which is the first-ever detection of bow shocks in the LMC. Orientation of the bow shock suggests that BI 237 was ejected from the OB association LH 82 (located at $\simeq 120 \mathrm{pc}$ in projection from the star). A by-product of our search is the detection of bow shocks generated by four OB stars in the field of the LMC and an arc-like structure attached to the candidate luminous blue variable R81 (HD 269128). The geometry of two of these bow shocks is consistent with the possibility that their associated stars were ejected from the 30 Doradus star-forming complex. We discuss implications of our findings for the problem of the origin of runaway stars and the early dynamical evolution of star clusters.
\end{abstract}

Key words. stars: kinematics and dynamics - stars: individual: BI 237 - open clusters and associations: individual: LH 82 open clusters and associations: individual: R136 (HD 38268) - stars: individual: HD 269128

\section{Introduction}

Although the majority of massive stars are situated in their parent clusters and $\mathrm{OB}$ associations, a significant population of young massive stars exists in the field, some of which are separated by hundreds of parsecs from known clusters and $\mathrm{OB}$ associations (Garmany et al. 1982; Garmany 1990; Massey \& Conti 1983; Massey et al. 1995). Some Galactic field stars have high measured peculiar (either radial or transverse) velocities and are therefore most likely runaway stars ejected from a cluster (Blaauw 1961; Gies \& Bolton 1986; Stone 1991; Zinnecker 2003). A straightforward way to prove the runaway nature of the field $\mathrm{OB}$ stars is to use the available kinematic data on these stars to back-trace their orbits to parent clusters (e.g. Hoogerwerf et al. 2001). Schilbach \& Röser (2008) make extensive use of this approach to show that most Galactic field OB stars are formed in clusters. Alternatively, the runaway nature of the field OB stars can be proved via detection of their bow shocks - the natural attributes of supersonically moving objects (e.g. Baranov et al. 1971; Van Buren \& McCray 1988). The geometry of detected bow shocks would allow one to infer the direction of stellar motion (Van Buren et al. 1995), thereby determining the possible parent clusters even for those field OB stars whose proper motions are still not available or measured with a low significance (Gvaramadze \& Bomans 2008b; Gvaramadze et al. 2010b). It is therefore tempting to search for bow shocks around field OB stars in the Large Magellanic Cloud (LMC) where accurate proper motion measurements are difficult, while bow shocks can still be resolved with modern infrared telescopes.

In this paper we present the results of a search for bow shocks in the LMC using archival Spitzer Space Telescope data. Our prime goal was to detect bow shocks produced by isolated, very massive stars that have previously been qualified as runaways on the basis of their large peculiar radial velocities (Sect. 2). We discovered a bow shock associated with one of these stars, the $\mathrm{O} 2 \mathrm{~V}\left(\left(\mathrm{f}^{*}\right)\right)$ star BI 237. A by-product of our search is detection of bow shocks produced by several other isolated OB stars, and two of these stars are located around the 30 Doradus nebula (Sect. 3). Implications of our findings for the problem of the origin of runaway stars and the early dynamical evolution of star clusters are discussed in Sect. 4. We use a distance of $50 \mathrm{kpc}$ for the LMC (Gibson 2000) so that 1' corresponds to $\simeq 14 \mathrm{pc}$.

\section{Very massive field stars as runaways}

The study of the massive star population in the LMC by Massey et al. (1995) has shown that a large number of young very massive (O2-type) stars is located at $\sim 100-200 \mathrm{pc}$ in projection from star clusters and $\mathrm{OB}$ associations. This finding was interpreted as indicating that the field can produce stars as massive as those 
born in clusters (Massey et al. 1995). An obvious alternative to this interpretation is that the massive field stars were actually formed in a clustered environment and subsequently ejected from their birth sites via dynamical processes (e.g. Clarke \& Pringle 1992; Pflamm-Altenburg \& Kroupa 2006); i.e., the field massive stars are runaway stars (Walborn et al. 2002; Brandl et al. 2007). The large separations from the possible parent clusters and the young $(\sim 2 \mathrm{Myr})$ ages of the very massive field stars imply that their (transverse) velocities should be as high as $\sim 50-100 \mathrm{~km} \mathrm{~s}^{-1}$ (Walborn et al. 2002). The large offsets from the parent clusters and the high peculiar velocities are not unusual for Galactic massive runaway stars. For example, the O4 If star $\mathrm{BD}+43^{\circ} 3654$ ejected from the Cyg OB2 association is located at about $80 \mathrm{pc}$ from the core of the association (Comerón \& Pasquali 2007; Gvaramadze \& Bomans 2008a), while the heliocentric radial velocity of the star is offset by $56 \mathrm{~km} \mathrm{~s}^{-1}$ from the systemic velocity of Cyg OB2 (Kobulnicky et al. 2010), which with the transverse peculiar velocity of the star of $\simeq 40 \mathrm{~km} \mathrm{~s}^{-1}$ (Comerón \& Pasquali 2007) corresponds to the total peculiar velocity of $\simeq 70 \mathrm{~km} \mathrm{~s}^{-1}$.

The runaway interpretation of the massive field stars in the LMC received strong support after the discovery that some of them have high peculiar radial velocities, much greater than the systemic velocity of the LMC (Massey et al. 2005; Evans et al. 2006, 2010). Earlier, Nota et al. (1994) and Danforth \& Chu (2001) had found that the systemic velocity of the candidate luminous blue variable S119 (HD 269687) and its circumstellar nebula is $\sim 100 \mathrm{~km} \mathrm{~s}^{-1}$ lower than that of the LMC, so they suggested that $\mathrm{S} 119$ could be a runaway star.

To prove the runaway nature of the field massive stars in the LMC unambiguously, it is necessary to ascertain their parent clusters. The proper motion measurements cannot help solve this problem in the near future. At the distance of the LMC, the transverse velocity of $\sim 50-100 \mathrm{~km} \mathrm{~s}^{-1}$ corresponds to a proper motion of $\sim 0.2-0.4 \mathrm{mas} \mathrm{yr}^{-1}$, which is too low to be measured with high confidence using the ground-based observations. The simpler solution is to infer the direction of stellar motion via the geometry of bow shocks produced by runaway stars (Gvaramadze \& Bomans 2008b) or, in the case of evolved massive runaway stars, through the asymmetry in the brightness distribution of associated circumstellar nebulae (Danforth \& Chu 2001; Gvaramadze et al. 2009). It is worth noting that two very massive Galactic runaway stars, $\mathrm{BD}+43^{\circ} 3654$ and $\lambda$ Cep (O6 I(n)f; Walborn 1973), are both associated with spectacular bow shocks (Comerón \& Pasquali 2007; Van Buren et al. 1995). One can therefore expect that some of the field stars in the LMC will manifest themselves in such secondary attributes of highvelocity runaway stars.

\section{Search for bow shocks in the LMC}

To search for bow shocks, we selected four isolated massive stars with high peculiar radial velocities (Massey et al. 2005; Evans et al. 2006, 2010) and added two isolated O2-type stars, BI 253 $(\mathrm{O} 2 \mathrm{~V}((\mathrm{f} *)))$ and $\mathrm{Sk}-68^{\circ} 137$ (O2 III(f*)), which Walborn et al. (2002) suggests are runaways owing to their large separation from their plausible birthplace in the central cluster, R136 (HD 38268), of the 30 Doradus nebula. The details of these stars (listed in order of their RA) are summarized in Table 1. For the first four stars, we give their peculiar radial velocities, while the transverse velocities are listed for the remaining two, inferred under the assumption that both stars were ejected 2 Myr ago from R136 (Walborn et al. 2002).
Table 1. Summary of candidate runaway stars in the LMC.

\begin{tabular}{lcc}
\hline \hline Star & Spectral type & $v\left(\mathrm{~km} \mathrm{~s}^{-1}\right)$ \\
\hline N11-026 & O2.5 III(f*) & $\simeq 35^{a}$ \\
Sk $-67^{\circ} 22$ & O2 If* & $\simeq 150^{b}$ \\
BI 237 & O2 V $\left(\left(f^{*}\right)\right)$ & $\simeq 120^{b}$ \\
30 Dor 016 & O2 III-If* & $\simeq 85^{c}$ \\
BI 253 & O2 V $\left(\left(f^{*}\right)\right)$ & $\sim 55^{d}$ \\
Sk $-68^{\circ} 137$ & O2 III (f*) & $\sim 100^{d}$ \\
\hline
\end{tabular}

Notes. ${ }^{(a)}$ Evans et al. (2006). ${ }^{(b)}$ Massey et al. (2005). ${ }^{(c)}$ Evans et al. (2010). ${ }^{(d)}$ Walborn et al. (2002).

From our experience in the search for bow shocks produced by $\mathrm{OB}$ stars ejected from Galactic star clusters (Gvaramadze \& Bomans 2008a,b; Gvaramadze et al. 2010b) using the archival data from the Midcourse Space Experiment (MSX) satellite (Price et al. 2001) and the Spitzer Space Telescope (Werner et al. 2004), we know that the bow shocks are visible mostly in $21.3 \mu \mathrm{m}$ (MSX band E) images and $24 \mu \mathrm{m}$ images obtained with the Multiband Imaging Photometer for Spitzer (MIPS; Rieke et al. 2004). The resolution of Spitzer $24 \mu \mathrm{m}$ images $\left(\sim 6^{\prime \prime}\right)$ is three times better than those of the MSX, so that in the search for bow shocks in the LMC we utilized the MIPS data alone.

The typical (transverse) size of bow shocks generated by Galactic OB stars (i.e. the extent of a bow shock in the direction perpendicular to the vector of the stellar motion) is several parsecs; e.g. the size of the bow shocks associated with the abovementioned massive runaway stars, $\mathrm{BD}+43^{\circ} 3654$ and $\lambda \mathrm{Cep}$, is $\simeq 5.0$ and $2.3 \mathrm{pc}$, respectively. If placed at the distance of the LMC, these bow shocks will have an angular size of $\simeq 10^{\prime \prime}-20^{\prime \prime}$, which is comparable to or several times greater than the angular resolution of the MIPS $24 \mu \mathrm{m}$ images. Thus, the bow shocks in the LMC can be resolved with the Spitzer imaging data!

Visual inspection of MIPS $24 \mu \mathrm{m}$ images $^{1}$ of fields containing our programme stars revealed a bow shock associated with only one of them, namely BI 237 . The non-detection of bow shocks around the remaining five programme stars is consistent with the observational fact that only a small fraction ( $\$ 20$ per cent) of runaway OB stars produce (observable) bow shocks (Gvaramadze \& Bomans 2008b and references therein).

Figure 1 gives an overview of the region northwest of BI 237 with two associations, LH 88 and LH 82, whose centres are separated in projection by $\simeq 65$ and 120 pc from the star. (The approximate boundaries of the associations are indicated by dashed circles; Bica et al. 1999.) The orientation of the bow shock generated by BI 237 (see Fig. 2) suggests that the more likely parent association of the star is LH 82 . LH 82 contains another very massive star, Sk $-67^{\circ} 211$ (O2 III(f*); Walborn et al. 2004). Assuming that BI 237 was indeed ejected from LH 82 and given the young $(\sim 2 \mathrm{Myr})$ age of the star, one finds that its transverse velocity should be $\sim 60 \mathrm{~km} \mathrm{~s}^{-1}$ if the star escaped from the core of LH 82 soon after the birth or higher if the ejection event occurred later on, so that the total peculiar velocity of the star is $\geq 130 \mathrm{~km} \mathrm{~s}^{-1}$. The angular size of the bow shock of $\simeq 20^{\prime \prime}$ corresponds to the linear size of $\simeq 4.8$ pc, i.e., a figure typical of massive runaway stars (see above). Using these estimates, one can constrain the number density of the ambient interstellar medium, $n_{\text {ISM. }}$. For the characteristic (transverse) size of a (parabolic) bow

\footnotetext{
1 The images, obtained in the framework of the Spitzer Survey of the Large Magellanic Cloud (Meixner et al. 2006), were retrieved from the NASA/IPAC Infrared Science Archive (http: //irsa .ipac. caltech.edu).
} 


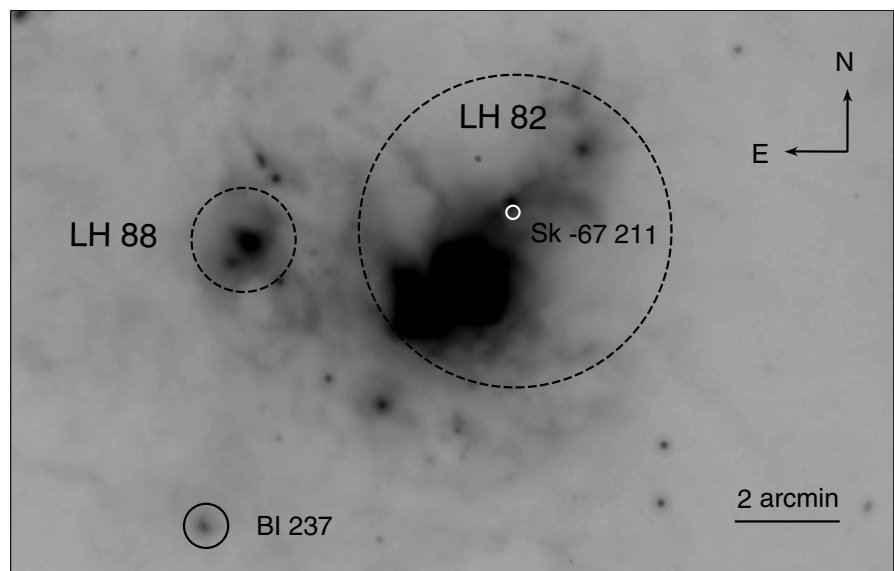

Fig. 1. MIPS $24 \mu \mathrm{m}$ image of the associations LH 82 and LH 88 (indicated by dashed circles). The position of the $\mathrm{O} 2 \mathrm{~V}\left(\left(\mathrm{f}^{*}\right)\right)$ star BI 237 and its bow shock are marked by a black solid circle, while the position of the $\mathrm{O} 2 \mathrm{III}\left(\mathrm{f}^{*}\right)$ star Sk $-67^{\circ} 211$ in $\mathrm{LH} 82$ is indicated by a white circle. At the distance of the LMC, $1^{\prime}$ corresponds to $\simeq 14 \mathrm{pc}$.

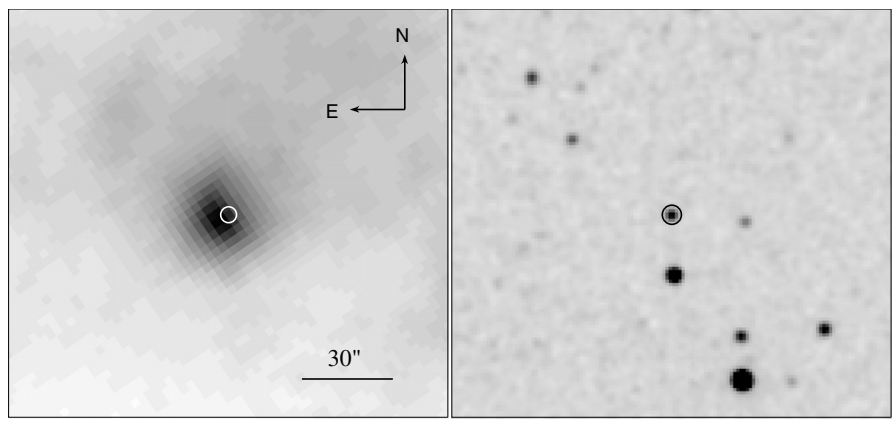

Fig. 2. Left: MIPS $24 \mu \mathrm{m}$ image of the bow shock associated with the $\mathrm{O} 2 \mathrm{~V}\left(\left(\mathrm{f}^{*}\right)\right)$ star BI 237. The position of BI 237 is marked by a circle. Right: 2MASS $J$ band image of the same field.

Table 2. Details of bow shock-producing stars.

\begin{tabular}{lcc}
\hline \hline Star & Spectral type & Association \\
\hline Sk $-66^{\circ} 16$ & O9.7 Ib $^{a}$ & KMHK 268 \\
Sk $-68^{\circ} 86$ & B1 $:^{b}$ & [SL63] 495 \\
Sk $-69^{\circ} 206$ & B2 $:^{b}$ & 30 Doradus \\
Sk $-69^{\circ} 288$ & B $0.5^{b}$ & 30 Doradus? \\
\hline
\end{tabular}

Notes. ${ }^{(a)}$ Evans et al. (2006). ${ }^{(b)}$ Rousseau et al. (1978).

shock of $\sim 2 \sqrt{2} R_{0}$, where $R_{0}=\left(\dot{M} v_{\infty} / 4 \pi \rho_{\text {ISM }} v_{\star}^{2}\right)^{1 / 2}$ is the standoff distance of the bow shock, $\dot{M}$ and $v_{\infty}$ are the mass-loss rate and terminal velocity of the stellar wind, $\rho_{\mathrm{ISM}}=1.4 m_{\mathrm{H}} n_{\mathrm{ISM}}$ is the density of the interstellar medium, $m_{\mathrm{H}}$ the mass of the hydrogen atom, and $v_{*}\left(\geq 130 \mathrm{~km} \mathrm{~s}^{-1}\right)$ the velocity of the star relative to the ambient medium, and using the wind parameters of BI 237, $\dot{M}=7.8 \times 10^{-7} M_{\odot} \mathrm{yr}^{-1}$ and $v_{\infty}=3400 \mathrm{~km} \mathrm{~s}^{-1}$ (Mokiem et al. 2007), one finds $n_{\text {ISM }} \leq 0.1 \mathrm{~cm}^{-3}$, i.e., a reasonable number.

A by-product of our search is the detection of bow shocks associated with four other OB stars in the field of the LMC (Fig. 3). The details of these stars are given in Table 2. The spectral types of the stars were found using the VizieR catalogue access tool ${ }^{2}$. The last column gives the possible birthplaces of the stars.

The bow shock produced by $\mathrm{Sk}-69^{\circ} 206$ is located at $\simeq 17^{\prime}$ $(\simeq 240 \mathrm{pc})$ to the west of R136 - the central cluster of the

${ }^{2}$ http://webviz.u-strasbg.fr/viz-bin/VizieR

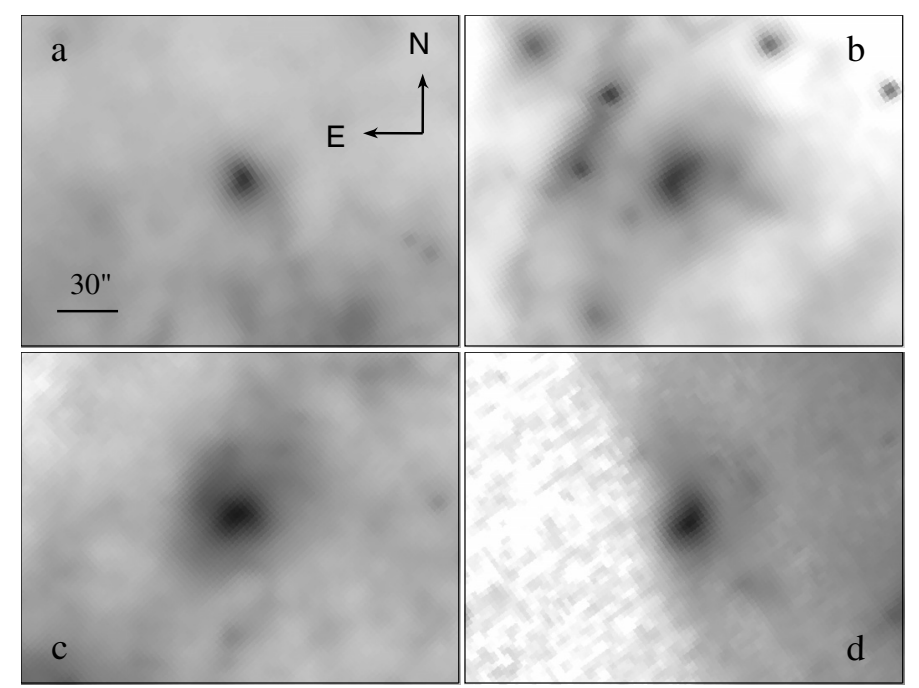

Fig. 3. MIPS $24 \mu \mathrm{m}$ images of bow shocks associated with a) $\mathrm{Sk}-69^{\circ}$ 206 , b) Sk $-69^{\circ} 288$, c) $\mathrm{Sk}-66^{\circ} 16$, and d) $\mathrm{Sk}-68^{\circ} 86$. The orientation and the scale of the images are the same.

30 Doradus nebula (Fig. 4). The orientation of the bow shock is consistent with the possibility that the associated star was ejected from the 30 Doradus nebula. Assuming that the ejection event occurred $\sim 2$ Myr ago, one finds the transverse velocity of $\mathrm{Sk}-69^{\circ} 206$ of $120 \mathrm{~km} \mathrm{~s}^{-1}$, which is comparable to the peculiar velocities of the candidate runaway stars in the LMC (see Table 1).

The bow shock produced by $\mathrm{Sk}-69^{\circ} 288$ is situated (at least in projection) within the association LH 113. The geometry of the bow shock suggests that $\mathrm{Sk}-69^{\circ} 288$ is moving away from the 30 Doradus nebula, which is located at $\simeq 21^{\prime}(\simeq 300 \mathrm{pc})$ to the west of the star (Fig. 4). Although we have no arguments against the association between $\mathrm{Sk}-69^{\circ} 288$ and LH 113, one cannot exclude the possibility that the actual birthplace of the star is the 30 Doradus nebula. In this connection, it is worth noting that the O5 V star ALS 19631 (Hanson 2003) was suggested as a member of the Cyg OB2 association on the basis of its location within the confines of the association (Comerón et al. 2002). The astrometric data on ALS 19631 and the geometry of the bow shock generated by this star, however, suggest that this runaway was instead ejected from the open cluster NGC6913 centred $\simeq 3^{\circ} .4$ west of the star (Gvaramadze \& Bomans 2008a).

The bow shocks associated with $\mathrm{Sk}-66^{\circ} 16$ and $\mathrm{Sk}-68^{\circ} 86$ are located, respectively, at $\sim 3.5^{\prime}(\simeq 50 \mathrm{pc})$ and $\simeq 2.5^{\prime}(\simeq 35 \mathrm{pc})$ from the clusters KMHK 268 (Fig. 5) and [SL63] 495 (Fig. 6). $\mathrm{Sk}-66^{\circ} 16$ is located in the N11 star-forming region, not far from our programme star N11-026 (see Fig. 5). We note the detection of a bow shock-like structure associated with one of the most massive stars in N11, the ON2 IIIf*: star N11-031 (Evans et al. 2006). This structure is facing towards the centre of the parent association LH 10 (Fig. 7a). Interestingly, the radial velocity of N11-031 is $\simeq 30 \mathrm{~km} \mathrm{~s}^{-1}$ greater than the median velocity of stars in N11 (Evans et al. 2006), which could be considered as indicating that this star is a runaway as well.

For the sake of completeness, we note also the detection of an arc-like nebula (Fig. 7b) attached to the candidate luminous blue variable R81 (HD 269128; Wolf et al. 1981; van Genderen 2001; cf. Gvaramadze et al. 2010a) and the $24 \mu \mathrm{m}$ counterpart to the circumstellar nebula around the O9f star Sk $-69^{\circ} 279$ (Weis et al. 1997). 


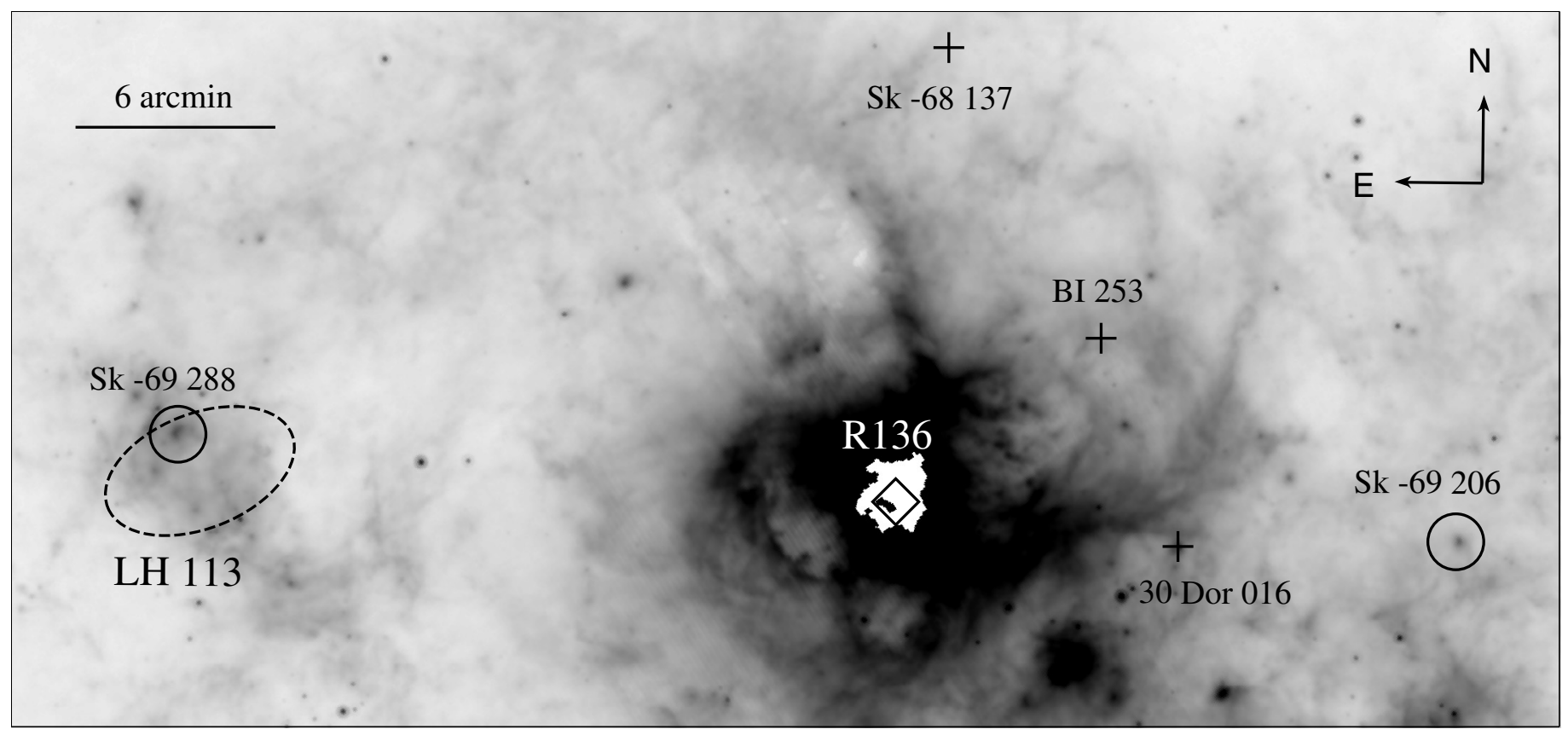

Fig. 4. MIPS $24 \mu \mathrm{m}$ image of the 30 Doradus nebula and its surroundings. The positions of Sk $-69^{\circ} 206$ and Sk $-69^{\circ} 288$ and their bow shocks are marked by solid circles. The positions of the three programme stars, Sk $-68^{\circ} 137$, BI 253, and 30 Dor 016, are marked by crosses. The diamond point shows the position of R136. The dashed ellipsoid indicates the approximate boundary of the association LH 113 .

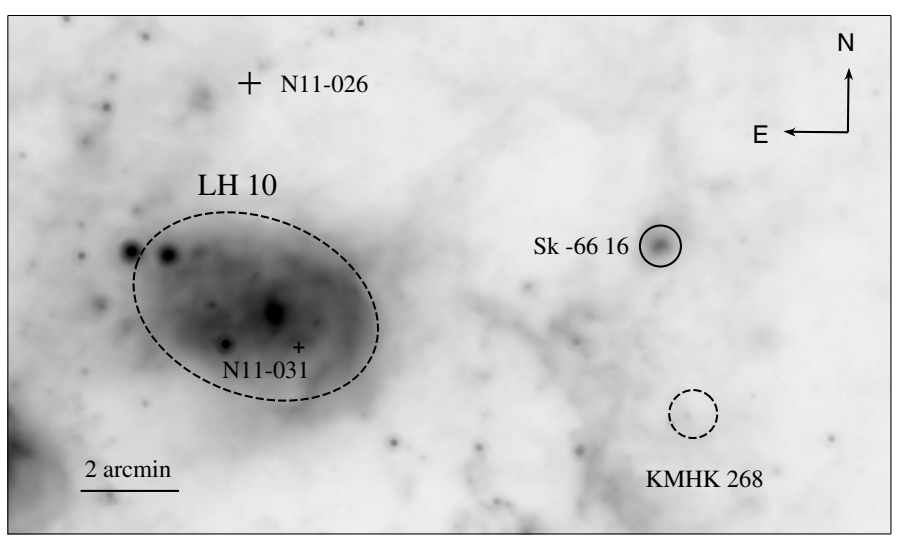

Fig. 5. MIPS $24 \mu \mathrm{m}$ image of the N11 star-forming region with the programme star N11-026 marked by a large cross. The small cross indicates the position of the ON2 IIIf*: star N11-031. The solid circle shows the position of $\mathrm{Sk}-66^{\circ} 16$ and its bow shock. The positions of the association LH 10 and the cluster KMHK 268 are indicated by a dashed ellipse and a dashed circle, respectively.

\section{Discussion and conclusion}

The discovery of a bow shock produced by BI 237 lends strong support to the idea that this and other isolated massive stars in the field of the LMC are runaway stars (Walborn et al. 2002; Massey et al. 2005; Evans et al. 2006, 2010). The young ages ( 2 Myr) of BI 237 and other O2-type field stars decidedly argue that their peculiar velocities cannot be explained by supernova explosions in binary systems (Blaauw 1961); the massive companion (primary) stars would simply have no time to end their lives in supernovae. Moreover, the high (measured or inferred) peculiar velocities of these stars cannot be accounted for within the framework of the binary-supernova scenario since it requires that the stellar supernova remnant (a 5-10 $M_{\odot}$ black hole) receive an unrealistically high $\left(\gtrsim 200-300 \mathrm{~km} \mathrm{~s}^{-1}\right)$ kick

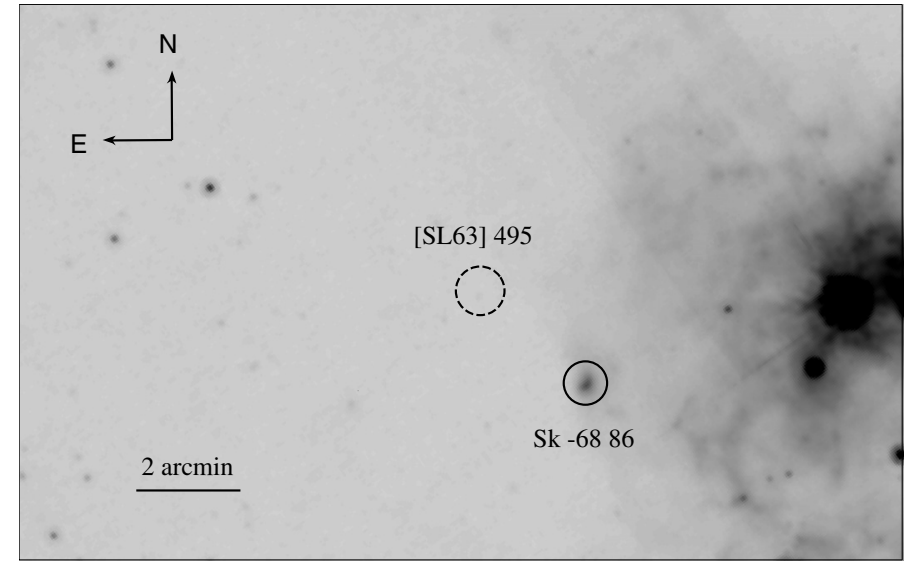

Fig. 6. MIPS $24 \mu \mathrm{m}$ image of the bow shock associated with $\mathrm{Sk}-68^{\circ} 86$ (marked by a solid circle). The position of the cluster [SL63] 495 is indicated by a dashed circle.

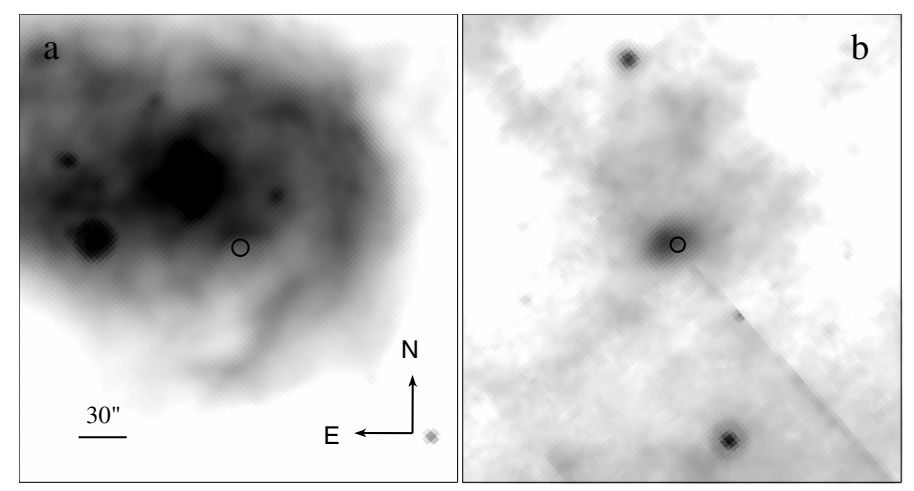

Fig. 7. MIPS $24 \mu \mathrm{m}$ images of a) a bow shock-like structure associated with N11-031 and b) an arc-like nebula attached to the candidate luminous blue variable R81 (HD 269128). The positions of both stars are indicated by circles. The orientation and the scale of the images are the same. 
velocity at birth (Gvaramadze \& Bomans 2008a; cf. Gvaramadze 2009). The only viable alternative is that the massive stars were ejected in the field via dynamical three- or four-body encounters (Poveda et al. 1967; Leonard \& Duncan 1990; Kroupa 1998; Pflamm-Altenburg \& Kroupa 2006; Gvaramadze, Gualandris \& Portegues Zwart 2008, 2009). Naturally, less massive (late B-type) stars are also ejected from their birth clusters by dynamical interactions (e.g. Kroupa 1998), but they would be difficult to observe in the LMC.

The large separation of some of the O2-type field stars from their plausible birthplaces implies that these stars were ejected soon after birth (which also argues against the binary-supernova scenario). This implication has an important consequence for understanding the early dynamical evolution of star clusters since it suggests that mass segregation in young clusters (the necessary condition for effective production of runaway $\mathrm{OB}$ stars) should be primordial rather than caused by the Spitzer instability. For example, R136 was found to already be mass-segregated at its age of about 2 Myr or younger (Campbell et al. 1992; Hunter et al. 1995; Brandl et al. 1996; de Grijs et al. 2002). But the Spitzer instability could be a very fast ( $\$ 0.5 \mathrm{Myr})$ process if the birth cluster is very dense (e.g. Kroupa 2008). Highprecision proper motion measurements for the massive field stars are therefore required to determine the timing of their ejections, thereby distinguishing between the primordial and the dynamical origins of mass segregation in young clusters (Gvaramadze \& Bomans 2008b). Future proper motion measurements with the space astrometry mission Gaia will allow us to solve this problem. At the same time, $N$-body experiments are required to quantify the expected differences between the two types of mass segregation in terms of the ejection of massive stars.

To conclude, the search for bow shocks in star-forming regions and subsequent identification of their associated stars serve as a useful tool for detecting runaway OB stars (e.g. Gvaramadze \& Bomans 2008b; Gvaramadze et al. 2010b), hence for constraining the dynamical evolution of their parent clusters. Further search for bow shocks around young massive clusters and OB associations in the LMC (when necessary, accompanied by followup spectroscopy of their associated stars) is therefore warranted.

Acknowledgements. We are grateful to S. Röser, H. Zinnecker, and the anonymous referee for carefully reading the manuscript and for useful comments, allowing us to improve the presentation of the paper. V.V.G. acknowledges financial support from the Deutsche Forschungsgemeinschaft. This research has made use of the NASA/IPAC Infrared Science Archive, which is operated by the Jet Propulsion Laboratory, California Institute of Technology, under contract with the National Aeronautics and Space Administration, the SIMBAD database, and the VizieR catalogue access tool, both operated at CDS, Strasbourg, France.

\section{References}

Baranov, V. B., Krasnobaev, K. V., \& Kulikovskii, A. G. 1971, Soviet Phys. Doklady, 15, 791

Bica, E. L. D., Schmitt, H. R., Dutra, C. M., \& Oliveira, H. L. 1999, AJ, 117, 238

Blaauw, A. 1961, Bull. Astron. Inst. Netherlands, 15, 265
Brandl, B. R., Sams, B. J., Bertoldi, F., et al. 1996, ApJ, 466, 254

Brandl, B. R., Portegies Zwart, S. F., Moffat, A. F. J., \& Chernoff, D. F. 2007, in Massive Stars in Interactive Binaries, ed. N. St.-Louis, \& A. F. J. Moffat (San Francisco: ASP), 629

Campbell, B., Hunter, D. A., Holtzman, J. A., et al. 1992, AJ, 104, 1721

Clarke, C. J., \& Pringle, J. E. 1992, MNRAS, 255, 423

Comerón, F., \& Pasquali, A. 2007, A\&A, 467, 23

Comerón, F., Pasquali, A., Rodighiero, G., et al. 2002, A\&A, 389, 874

Danforth, C. W., \& Chu, Y.-H. 2001, ApJ, 552, L155

de Grijs, R., Johnson, R. A., Gilmore, G. F., \& Frayn, C. M. 2002, MNRAS, 331, 228

Evans, C. J., Lennon, D. J., Smartt, S. J., \& Trundle, C. 2006, A\&A, 456, 623

Evans, C. J., Walborn, N. R., Crowther, P. A., et al. 2010, ApJ, 715, L74

Garmany, C. D. 1990, in Properties of Hot Luminous Stars (San Francisco: ASP), 16

Garmany, C. D., Conti, P. S., \& Chiosi, C. 1982, ApJ, 263, 777

Gibson, B. K. 2000, MmSAI, 71, 693

Gies, D. R., \& Bolton, C. T. 1986, ApJS, 61, 419

Gvaramadze, V. V. 2009, MNRAS, 395, L85

Gvaramadze, V. V., \& Bomans, D. J. 2008a, A\&A, 485, L29

Gvaramadze, V. V., \& Bomans, D. J. 2008b, A\&A, 490, 1071

Gvaramadze, V. V., Gualandris, A., \& Portegies Zwart, S. 2008, MNRAS, 385, 929

Gvaramadze, V. V., Gualandris, A., \& Portegies Zwart, S. 2009, MNRAS, 400, 524

Gvaramadze, V. V., Fabrika, S., Hamann, W.-R., et al. 2009, MNRAS, 400, 524

Gvaramadze, V. V., Kniazev, A. Y., \& Fabrika, S. 2010a, MNRAS, 405, 1047

Gvaramadze, V. V., Kniazev, A. Y., Hamann, W.-R., et al. 2010b, MNRAS, 403, 760

Hanson, M. M. 2003, ApJ, 597, 957

Hoogerwerf, R., de Bruijne, J. H. J., \& de Zeeuw, P. T. 2001, A\&A, 365, 49

Hunter, D. A., Shays, E. J., Holtzman, J. A., et al. 1995, ApJ, 448, 179

Kobulnicky, H. A., Gilbert, I. J., \& Kiminki, D. C. 2010, ApJ, 710, 549

Kroupa, P. 1998, MNRAS, 298, 231

Kroupa, P. 2008, in The Cambridge $N$-Body Lectures, ed. S. J. Arseth, C. A.

Tout, \& R. A. Mardling (Berlin: Springer), Lect. Notes Phys., 760, 181

Leonard, P. J. T., \& Duncan, M. J. 1990, AJ, 99, 608

Massey, P., \& Conti, P. S. 1983, ApJ, 273, 576

Massey, P., Lang, C. C., Degioia-Eastwood, K., \& Garmany, C. D. 1995, ApJ, 438, 188

Massey, P., Puls, J., Pauldrach, A. W. A., et al. 2005, ApJ, 627, 477

Meixner, M., Gordon, K. D., Indebetouw, R., et al. 2006, AJ, 132, 2268

Mokiem, M. R., de Koter, A., Evans, C. J., et al. 2007, A\&A, 465, 1003

Nota, A., Drissen, L., Clampin, M., et al. 1994, in Circumstellar Media in the Late Stages of Stellar Evolution, ed. R. E. S. Clegg, I. R. Stevens, \& W. P. S.

Miekle (Cambridge: Cambridge Univ. Press), 89

Pflamm-Altenburg, J., \& Kroupa, P. 2006, MNRAS, 373, 295

Poveda, A., Ruiz, J., \& Allen, C. 1967, Bol. Obs. Tonantzintla Tacubaya, 4, 86

Price, S. D., Egan, M. P., Carey, S. J., Mizuno, D. R., \& Kuchar, T. A. 2001, AJ, 121,2819

Rieke, G. H., Young, E. T., Engelbracht, C. W., et al. 2004, ApJS, 154, 25

Rousseau, J., Martin, N., Prévot, L., et al. 1978, A\&AS, 31, 243

Schilbach, E., \& Röser, S. 2008, A\&A, 489, 105

Stone, R. C. 1991, AJ, 102, 333

Van Buren, D., \& McCray, R. 1988, ApJ, 329, L93

Van Buren, D., Noriega-Crespo, A., \& Dgani, R. 1995, AJ, 110, 2914

van Genderen, A. M. 2001, A\&A, 366, 508

Walborn, N. R. 1973, AJ, 78, 1067

Walborn, N. R., Howarth, I. D., Lennon, D. J., et al. 2002, AJ, 123, 2754

Walborn, N. R., Morrell, N. I., Howarth, I. D., et al. 2004, ApJ, 608, 1028

Weis, K., Chu, Y.-H., Duschl, W. J., \& Bomans, D. J. 1997, A\&A, 325, 1157

Werner, M. W., Roellig, T. L., Low, F. J., et al. 2004, ApJS, 154, 1

Wolf, B., Stahl, O., de Groot, M. J. H., \& Sterken, C. 1981, A\&A, 99, 351

Zinnecker, H. 2003, in A Massive Star Odyssey: From Main Sequence to Supernova, ed. K. van der Hucht, A. Herrero, \& C. Esteban, IAU Symp., 212,80 